\title{
Dual-energy contrast-enhanced digital mammography: initial clinical results
}

\author{
Clarisse Dromain • Fabienne Thibault • Serge Muller • \\ Françoise Rimareix • Suzette Delaloge • \\ Anne Tardivon • Corinne Balleyguier
}

Received: 23 April 2010 /Revised: 30 July 2010 / Accepted: 30 July 2010 / Published online: 14 September 2010

(C) European Society of Radiology 2010

\begin{abstract}
Objective To assess the diagnostic accuracy of Dual-Energy Contrast-Enhanced Digital Mammography (CEDM) as an adjunct to mammography (MX) versus MX alone and versus mammography plus ultrasound (US).

Materials and methods 120 women with 142 suspect findings on MX and/or US underwent CEDM. A pair of low- and high-energy images was acquired using a modified full-field digital mammography system. Exposures were taken
\end{abstract}

C. Dromain · C. Balleyguier

Department of Imaging, Institut de Cancérologie Gustave-Roussy,

39, rue Camille Desmoulins,

94805 Villejuif Cedex, France

F. Thibault $\cdot$ A. Tardivon

Department of Imaging, Institut Curie,

26 rue d'Ulm,

75248 Cedex 05 Paris, France

S. Muller

GE Healthcare,

283 , rue de la minière,

78530 Buc, France

F. Rimareix

Department of Surgery, Institut Gustave-Roussy,

39, rue Camille Desmoulins,

94805 Villejuif Cedex, France

S. Delaloge

Department of Medicine, Institut Gustave-Roussy,

39, rue Camille Desmoulins,

94805 Villejuif Cedex, France

C. Dromain $(\bowtie)$

Department of Radiology,

Institut de Cancérologie Gustave-Roussy,

39, rue Camille Desmoulins,

94805 Villejuif Cedex, France

e-mail: dromain@igr.fr in $\mathrm{MLO}$ at $2 \mathrm{~min}$ and in $\mathrm{CC}$ at $4 \mathrm{~min}$ after the injection of $1.5 \mathrm{ml} / \mathrm{kg}$ of an iodinated contrast agent. One reader evaluated MX, US and CEDM images during 2 sessions 1 month apart. Sensitivity, specificity, and area under the ROC curve were estimated.

Results The results from pathology and follow-up identified 62 benign and 80 malignant lesions. Areas under the ROC curves were significantly superior for MX+CEDM than it was for MX alone and for MX+US using BI-RADS. Sensitivity was higher for MX+CEDM than it was for MX (93\% vs. $78 \%$; $p<0.001)$ with no loss in specificity. The lesion size was closer to the histological size for CEDM. All 23 multifocal lesions were correctly detected by MX+CEDM vs. 16 and 15 lesions by MX and US respectively.

Conclusion Initial clinical results show that CEDM has better diagnostic accuracy than mammography alone and mammography+ultrasound.

\section{Introduction}

Mammography is a well-established, cost-effective imaging technique for breast cancer detection [1-3]. Conventional mammography and (despite some progress) full-field digital mammography (MX) have limitations, particularly in dense [4] and treated breasts. Ultrasound is a complementary technique to mammography, especially for dense breasts [5] and is part of the standard of care in diagnostic procedures. Manual US examinations are time-consuming [6] operatordependant and findings have to be characterised during the procedure with limited possibility of a second independent evaluation of already captured images. Contrast-enhanced breast imaging techniques (like CT and MRI) are used for detection of angiogenesis by tracking contrast agent up-take and wash-out in tissues. Even if reported to be useful in the 
detection of breast carcinoma [7, 8], CT has the drawback of high radiation dose levels. Contrast-enhanced MRI seems to be currently the most sensitive breast cancer detection technique [9], but have high false positive rate and still carries the burden of higher costs and lower availability.

Taking advantage of the inherent capabilities of image processing in digital mammography, advanced applications may bring additional clinical and cost benefits to the current standard of care. Among these applications, contrast-enhanced digital mammography (CEDM) may specifically open the door to detection of angiogenesis in the mammography suite.

Clinical feasibility and initial experiences with CEDM have been reported for two techniques: temporal subtraction and dual energy [10-13]. Temporal subtraction CEDM involves a mask and a series of exposures, taken respectively before and after injection of contrast agent.

This article presents the initial clinical performance results of dual-energy CEDM as an adjunct to MX versus $\mathrm{MX}$ alone and versus MX+US.

\section{Materials and methods}

\section{Patients}

From March 2007 to March 2008, 120 patients with a mean age of 56 years (range 27-86 years) were enrolled in this prospective study. The study was approved by the Institutional Review Board and all patients gave their written informed consent. Inclusion criteria were recalls from screening with unresolved findings after mammography and ultrasound. Exclusion criteria were an isolated cluster of microcalcifications, pregnancy or possible pregnancy, or a history of allergy to an iodinated contrast agent. Suspect breast lesions included the association of mammographic and ultrasound abnormal findings in 81 patients, mammographic abnormal findings alone in 14 patients and ultrasound abnormal findings alone in 23 patients. Two patients had a palpable nodule with normal mammograms and ultrasound. Cytological or histopathological analysis of the suspect breast lesions were obtained by fine needle aspirations for 11 lesions, by core biopsy for 41 lesions and by surgery for 77 lesions. For the 13 lesions for which only cytological analyses were involved, 6- and 12-month follow-up mammography and ultrasound examinations were performed to confirm the diagnosis of a benign lesion.

Technique of dual-energy CEDM examination

All CEDM examinations were performed with an experimental device developed by GE Healthcare (Chalfont St. Giles, UK) allowing dual-energy CEDM acquisitions. It used a current full-field digital mammography system using a flat panel detector with CsI absorber, field size $19 \times 23$, del pitch of $100 \mathrm{~mm}$, image matrix size 1,914×2,294 (Senographe DS), with some specific software and hardware adaptations for acquisition and image processing. Indeed, the low energy exposures used in conventional mammography are not optimal for the visualisation of iodine. To increase the sensitivity to a low concentration of iodine with only a modest increase in the dose delivered to the patient, the x-ray spectrum must be changed to deliver energies just above the K-edge of iodine $(33.2 \mathrm{keV})$. The digital mammography system was modified accordingly by adding a copper filter specifically used for CEDM, in addition to the usual molybdenum and rhodium filters used for standard mammography. Moreover, a high voltage range of $45-49 \mathrm{kVp}$ was used (instead of $26-32 \mathrm{kVp}$ for conventional digital mammography) [14]. Typically, for a $5 \mathrm{~cm}$-thick, $50 \%$ glandular breasts, exposure times were around $1 \mathrm{~s}$ and $3 \mathrm{~s}$ for the low and high energy images, respectively.

A catheter was inserted into the antecubital vein of the arm contra-lateral to the breast of concern. A one-shot intravenous injection of $1.5 \mathrm{ml} /$ body weighted of non-ionic contrast agent (Xenetix 300, Guerbet France) was then performed, using a power injector (Medrad, Pittsburgh, PA, USA) at a rate of $3 \mathrm{ml} / \mathrm{s}$ with a bolus chaser. Two minutes after the initiation of the contrast agent administration the breast was compressed in an MLO view and a pair of lowand high-energy images was acquired. The breast was then compressed in the CC position and a new pair of low- and high-energy exposures was performed $4 \mathrm{~min}$ after the initiation of contrast agent administration. A combination of low-energy and high-energy images through a specific image processing [15] was performed in order to generate two subtracted images with contrast agent uptake information (one in the MLO and one in the CC view) (Fig. 1).

The total X-ray dose delivered to the patient for a pair of low- and high-energy images was estimated to be between 0.7 and $3.6 \mathrm{mGy}$ depending on breast thickness (30 to $80 \mathrm{~mm}$ ) and tissue composition (0 to $100 \%$ glandular tissue). This dose level corresponds to about 1.2 times the dose delivered for a standard digital mammogram. The average glandular dose(AGD) for the low energy image was equivalent to that of one conventional mammogram, while for high energy, it was approximately $20 \%$ of the dose of one conventional mammogram.

A simulation program was used to estimate AGD for the pair of low and high-energy images. The simulated values have been validated by comparison with reference values found in publications [16-18]. Concerning the AGD for the low-energy spectra, the differences between the simulated and reference values were $3 \%$ in average. For the highenergy spectra, the comparison of AGD for a Mo/Cu $49 \mathrm{kVp}$ spectrum gave approximately $5 \%$ relative difference. 


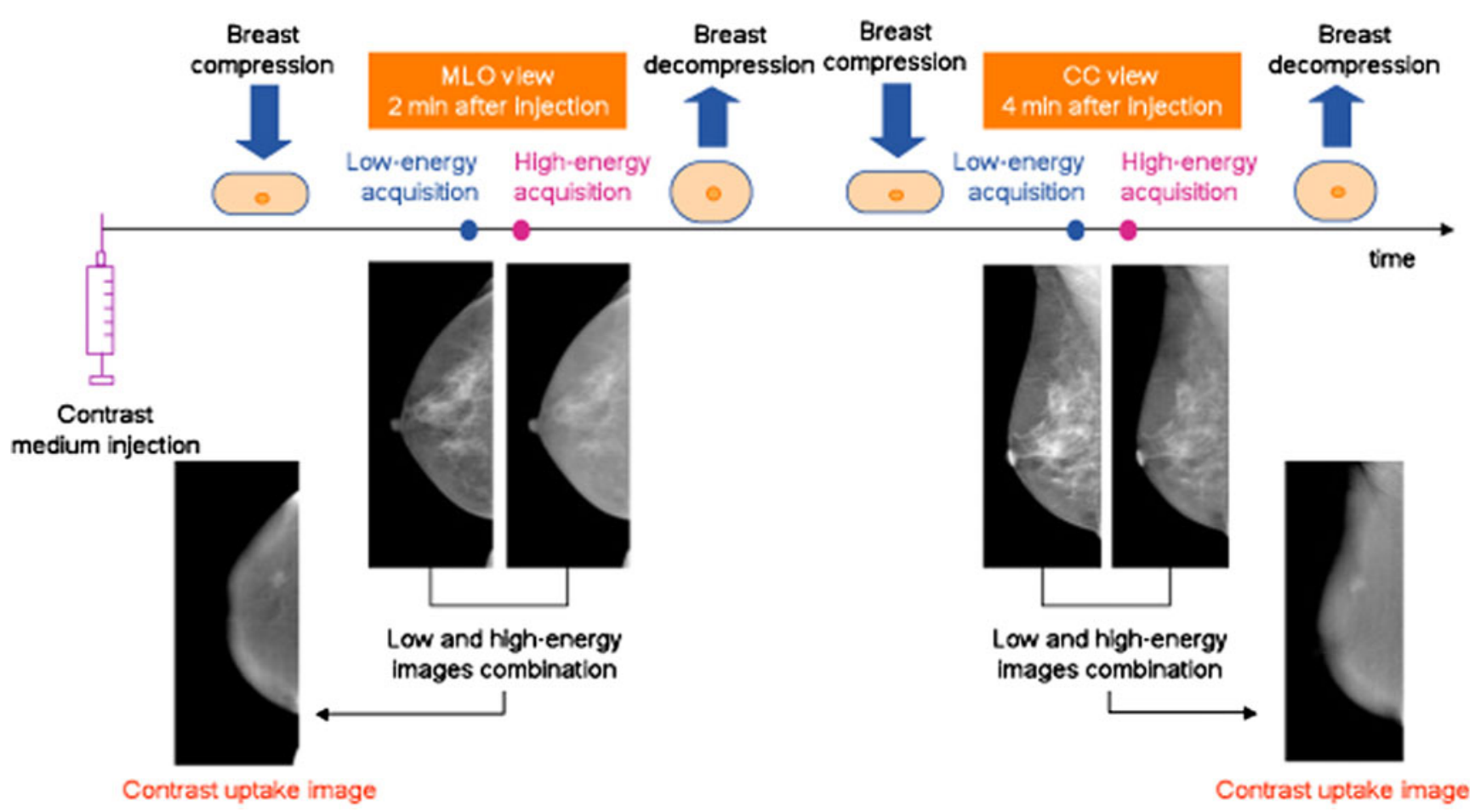

Fig. 1 Schema of the technique of dual-energy CEDM examination

Image analysis

A research workstation was used for image analysis. All images were retrospectively evaluated by one experienced breast radiologist (CD) with previous experience of CEDM examinations. Images were read blind to patient history, clinical information and truth. To minimise bias risks from remembering cases, there were two reading sessions separated by a time interval of 6 weeks. The first session included a review of MX alone and then MX+CEDM images and the second session included a review of MX+US images. For US examination reading only screening US images captured by the radiologist who performed US examination and recorded in the PACS (screen shots) were reviewed. The probability of malignancy (probability for a lesion to be malignant) using a 7-step scale and a BI-RADS 5-step assessment (BIRADS 0 excluded) were evaluated for each imaging technique (mammography, mammography+ultrasound and mammography+dual-energy CEDM) and each lesion. Breast density was evaluated on mammography using the BI-RADS criteria of the American College of Radiology. The type of findings depicted on mammography and US were reported according to the breast imaging lexicon from the American College of Radiology.

The subtracted CEDM images were reviewed using reading criteria based on contrast enhancement intensity and morphology. The following data were recorded for each tumour: presence or absence of contrast agent uptake, qualitative assessment of the intensity in 3 categories (strong, intermediate and weak), morphological assessment, size (longest axis), the location in the breast and consistency with the mammographic and US findings

\section{Statistical analysis}

The reference standard was abnormality described in all cases as being either benign or malignant. Three lesions with a diagnosis of precancerous tumour were classified as being benign for statistical analysis. The primary unit of analysis was the lesion. Bivariate binormal ROC curves were estimated and areas beneath them were compared using ROCKIT software. Sensitivity and specificity were estimated taking the probability of malignancy of 4 or higher and BI-RADS 4 or higher as positive test results. Comparisons of sensitivity or specificity between MX and MX+CEDM and MX+US were made using McNemar's chi-squared test with continuity correction. A comparison of the lesion sizes measured at mammography, ultrasound and CEDM was made with the histological size for respectively 59, 61 and 60 lesions.

\section{Results}

Breast tumours

There were 80 malignant, 50 benign and 3 pre-cancerous lesions ( 1 case of atypical hyperplasia and 2 cases of lobular carcinoma in situ). Malignant lesions were multifocal in 10 patients (bifocal in 7 patients and tri-focal in 3 patients). 
Table 1 Intensity of the CEDM enhancement

\begin{tabular}{lllllll}
\hline $\begin{array}{l}\text { Intensity of } \\
\text { enhancement }\end{array}$ & & $\begin{array}{l}\text { No } \\
\text { enhancement }\end{array}$ & $\begin{array}{l}\text { Very } \\
\text { weak }\end{array}$ & Weak & Moderate & High \\
\hline Malignant lesions $(n=80)$ & MLO 2 min. & 8 & 10 & 16 & 24 & 22 \\
& CC 4 min. & 10 & 0 & 10 & 24 & 36 \\
Benign lesions $(n=50)$ & MLO 2 min. & 37 & 1 & 3 & 8 & 1 \\
& CC 4 min. & 37 & 0 & 3 & 7 & 3 \\
Pre-cancerous lesions $(n=3)$ & MLO 2 min. & 0 & 0 & 1 & 1 & 1 \\
& CC 4 min. & 2 & 0 & 0 & 1 & 0 \\
\hline
\end{tabular}

$M L O$ mediolateral oblique view, $C C$ cranio-caudal view

circumscribed masses, 39 non-circumscribed masses without posterior acoustic features, 50 non-circumscribed margins with posterior shadowing.

CEDM Enhancement was observed in 74 out of $80(92 \%)$ malignant lesions. The enhancing lesions were 53 masses and 27 non-masses. Enhancement was not observed in 2 cases of ductal carcinoma in situ, 2 cases of tubular carcinoma and 2 cases of invasive ductal carcinoma (one grade 1 and one grade 2).

Enhancement was also observed in 13 of the $50(26 \%)$ benign lesions corresponding to 1 case of intraductal papilloma, 3 cases of fibroadenoma, 2 cases of radial scar with one associated with typical ductal hyperplasia, 2 cases of adenosis, 1 case of grade 1 phylloid tumour, 2 cases of cytosteatonecrosis and 2 cases of fibrocystic mastopathy.

The intensity levels of DE-CEDM enhancement for malignant and benign lesions are summarised in Table 1.

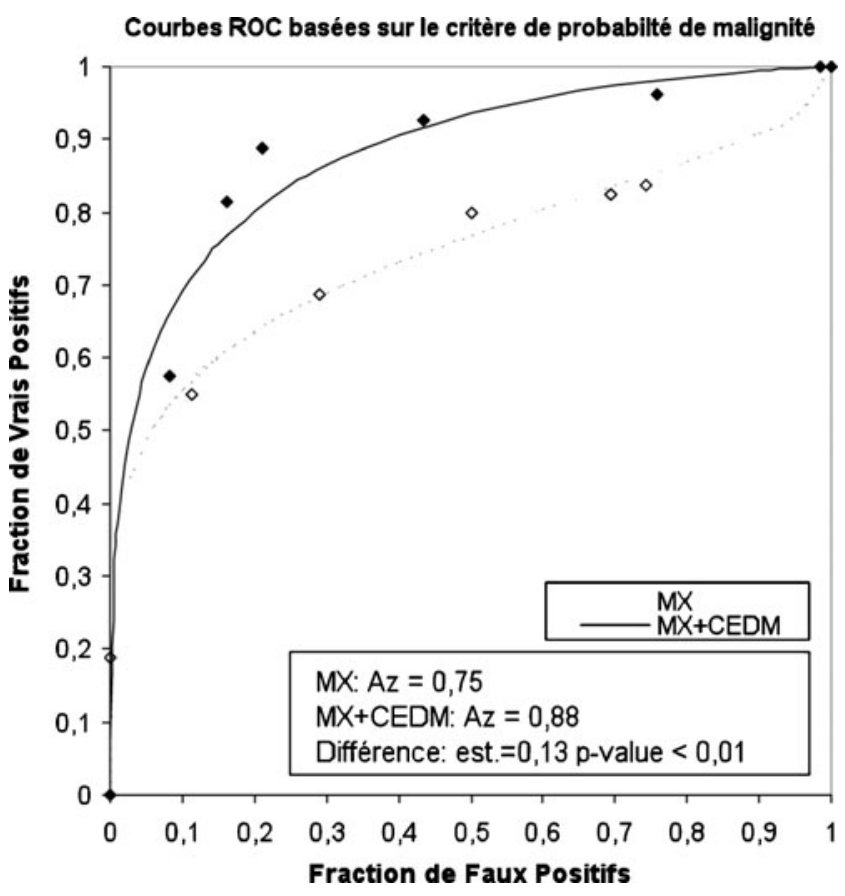

Fig. 2 Comparison of mammography and CEDM: ROC curves for the probability of malignancy

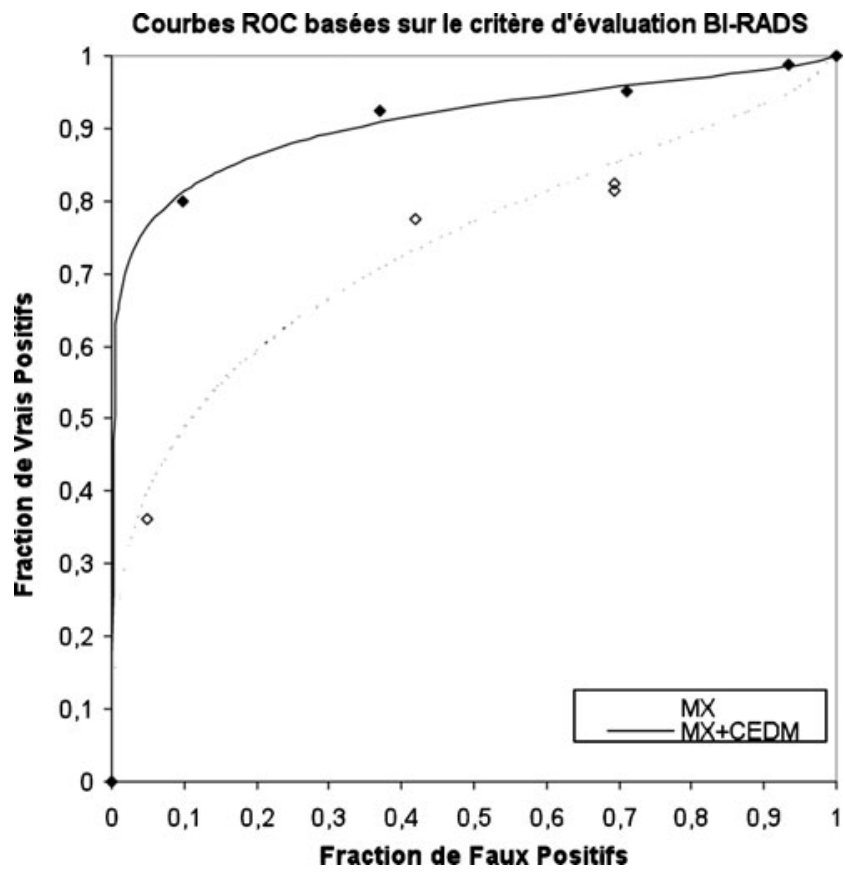

Fig. 3 Comparison of mammography and CEDM: ROC curves for BI-RADS assessment 
Table 2 Comparison of Mammography alone and $\mathrm{MX}+\mathrm{CEDM}$

$M X$ mammography, DE-CEDM dual-energy contrast-enhanced digital mammography, Est. estimate, $S E$ standard error, $C I$ confidence interval, $\operatorname{Pr}$. Mal. probability of malignancy

\begin{tabular}{|c|c|c|c|c|c|c|c|c|}
\hline & \multicolumn{2}{|l|}{ MX } & \multicolumn{2}{|c|}{$\mathrm{MX}+\mathrm{CEDM}$} & \multicolumn{4}{|c|}{ Difference } \\
\hline & Est. & SE & Est. & SE & Est. & SE & $95 \% \mathrm{CI}$ & $p$ value \\
\hline \multicolumn{9}{|c|}{ Area under ROC curve } \\
\hline Pr. Mal. & 0.75 & 0.04 & 0.88 & 0.03 & 0.13 & 0.04 & {$[0.05,0.20]$} & $<0.01$ \\
\hline BI-RADS & 0.74 & 0.04 & 0.91 & 0.02 & 0.17 & 0.04 & {$[0.08,0.23]$} & $<0.01$ \\
\hline \multicolumn{9}{|l|}{ Sensitivity } \\
\hline Pr. Mal. & 0.80 & 0.04 & 0.93 & 0.03 & 0.13 & 0.05 & {$[0.05,0.23]$} & 0.02 \\
\hline BI-RADS & 0.78 & 0.05 & 0.93 & 0.03 & 0.15 & 0.05 & {$[0.07,0.25]$} & $<0.01$ \\
\hline \multicolumn{9}{|l|}{ Specificity } \\
\hline Pr Mal. & 0.50 & 0.06 & 0.56 & 0.06 & 0.06 & 0.07 & {$[-0.09,0.22]$} & 0.50 \\
\hline BI-RADS & 0.58 & 0.06 & 0.63 & 0.06 & 0.05 & 0.06 & {$[-0.10,0.19]$} & 0.63 \\
\hline
\end{tabular}

Comparison of Mammography (MX), mammography+ ultrasound (MX+US) and MX+DE-CEDM

The area under the ROC curve was higher for MX+CEDM than it was for MX alone ( $p<0.01$ using either Probability of Malignancy or BI-RADS) (Figs. 2 and 3). Sensitivity was higher for MX+CEDM than it was for MX using either Probability of Malignancy $(93 \%$ vs $80 \% ; p<0.01)$ or BI-RADS (93\% vs $78 \% ; p<0.01)$ with no loss in specificity (Table 2) (Figs. 6 and 8).

The area under the ROC curve was also significantly higher for $\mathrm{MX}+\mathrm{CEDM}$ than it was for $\mathrm{MX}+\mathrm{US}$, for BI-RADS assessment (Figs. 4 and 5). Sensitivity and specificity of $\mathrm{MX}+\mathrm{CEDM}$ were not statistically different

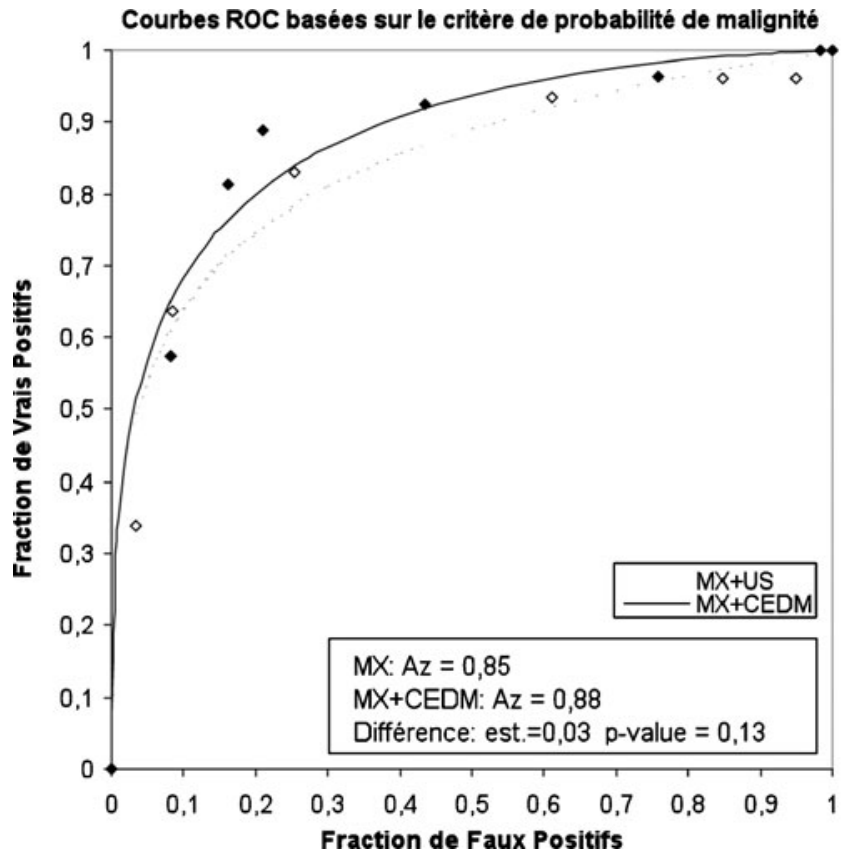

Fig. 4 Comparison of mammography+US and CEDM: ROC curves for the probability of malignancy versus $\mathrm{MX}+\mathrm{US}$ (Table 3). MX+CEDM also had higher positive and negative predictive values versus $\mathrm{MX}$ alone and versus $\mathrm{MX}+\mathrm{US}$ (Table 4).

The mean sizes of the lesions were measured as $14.3 \mathrm{~mm}, 10.8 \mathrm{~mm}$ and $16.1 \mathrm{~mm}$ respectively on $\mathrm{MX}$, US and MX+CEDM. MX+CEDM was the breast imaging method with the smallest difference in mean size and with no significant difference compared with histology (-0.7 mm, SE 1.5) (Table 5).

Among the ten patients with 23 multifocal histologically proven lesions, all were detected by MX+CEDM vs. 16 and 15 lesions detected by mammography alone and ultrasound respectively (Table 6).

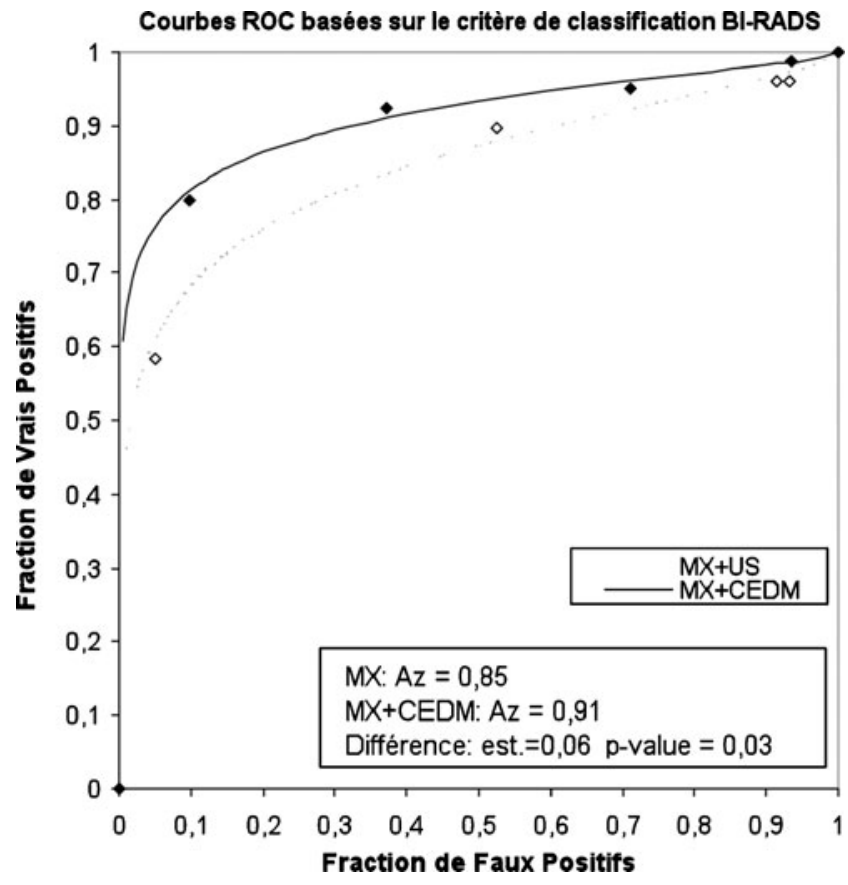

Fig. 5 Comparison of mammography+US and CEDM: ROC curves for BI-RADS assessment 
Table 3 Comparison of Mammography+ultrasound and $\mathrm{MX}+\mathrm{CEDM}$

$M X+U S$ mammography+ ultrasound, CEDM contrastenhanced digital mammography, Est. estimate, SE standard error, CI confidence interval, Pr. Mal., probability of malignancy

\begin{tabular}{|c|c|c|c|c|c|c|c|c|}
\hline & \multicolumn{2}{|c|}{$\mathrm{MX}+\mathrm{US}$} & \multicolumn{2}{|c|}{$\mathrm{MX}+\mathrm{CEDM}$} & \multicolumn{4}{|c|}{ Difference } \\
\hline & Est. & SE & Est. & $\mathrm{SE}$ & Est. & $\mathrm{SE}$ & $95 \% \mathrm{CI}$ & $p$ value \\
\hline \multicolumn{9}{|c|}{ Area under ROC curve } \\
\hline Pr. Mal. & 0.85 & 0.03 & 0.88 & 0.03 & 0.03 & 0.03 & {$[0.03 ; 0.1]$} & 0.13 \\
\hline BI-RADS & 0.85 & 0.04 & 0.91 & 0.03 & 0.06 & 0.03 & {$[0.00 ; 0.13]$} & 0.03 \\
\hline \multicolumn{9}{|l|}{ Sensitivity } \\
\hline Pr. Mal. & 0.94 & 0.03 & 0.92 & 0.03 & -0.02 & 0.03 & {$[0.10 ; 0.07]$} & 1 \\
\hline BI-RADS & 0.90 & 0.03 & 0.92 & 0.03 & 0.02 & 0.04 & {$[0.06 ; 0.10]$} & 0.72 \\
\hline \multicolumn{9}{|l|}{ Specificity } \\
\hline Pr Mal. & 0.39 & 0.06 & 0.56 & 0.06 & 0.17 & 0.08 & {$[0.00 ; 0.34]$} & 0.06 \\
\hline BI-RADS & 0.47 & 0.07 & 0.63 & 0.06 & 0.16 & 0.08 & {$[0.01 ; 0.32]$} & 0.08 \\
\hline
\end{tabular}

Table 4 Comparison of positive and negative predictive values with Mammography, Mammography+ultrasound and MX $+\mathrm{CEDM}$

\begin{tabular}{|c|c|c|c|c|c|c|c|c|c|}
\hline & \multicolumn{3}{|l|}{ MX } & \multicolumn{3}{|c|}{ MX+US } & \multicolumn{3}{|c|}{$\mathrm{MX}+\mathrm{CEDM}$} \\
\hline & Est & SE & $95 \% \mathrm{CI}$ & Est & $\mathrm{SE}$ & $95 \% \mathrm{CI}$ & Est & SE & $95 \% \mathrm{CI}$ \\
\hline \multicolumn{10}{|l|}{ PPV } \\
\hline Pr. Mal. & 0.67 & 0.05 & {$[0.57 ; 0.77]$} & 0.67 & 0.05 & {$[0.57 ; 0.75]$} & 0.73 & 0.04 & {$[0.64 ; 0.82]$} \\
\hline BI-RADS & 0.70 & 0.05 & {$[0.60 ; 0.78]$} & 0.69 & 0.05 & {$[0.59 ; 0.78]$} & 0.76 & 0.04 & {$[0.67 ; 0.84]$} \\
\hline \multicolumn{10}{|l|}{ NPV } \\
\hline Pr Mal. & 0.66 & 0.07 & {$[0.51 ; 0.79]$} & 0.82 & 0.07 & {$[0.63 ; 0.93]$} & 0.85 & 0.06 & {$[0.71 ; 0.94]$} \\
\hline BI-RADS & 0.67 & 0.06 & {$[0.53 ; 0.79]$} & 0.78 & 0.07 & {$[0.61 ; 0.90]$} & 0.87 & 0.05 & {$[0.73 ; 0.95]$} \\
\hline
\end{tabular}

$M X$ mammography, $U S$ ultrasound, $C E D M$ contrast-enhanced digital mammography, $P P V$ positive predictive value, $N P V$ negative predictive value, $P r$. Mal. probability of malignancy, $S E$ standard error, $C I$ confidence interval

\section{Discussion}

Among the clinical studies which have investigated CEDM, most have focused on the temporal subtraction method. In their study, Jong et al. have performed temporal CEDM on

Table 5 Comparison of the size of the lesions measured at MX, US and $\mathrm{MX}+\mathrm{CEDM}$ versus histology

\begin{tabular}{lccc}
\hline & MX & US & MX+CEDM \\
\hline Mean (mm) & 14.3 & 10.8 & 16.1 \\
Variance & 62.8 & 34.2 & 121.0 \\
Difference vs. histology & & & \\
Number of observations & 59 & 61 & 60 \\
Mean (mm) & -1.3 & -5.3 & -0.7 \\
Standard Error & 1.1 & 1.2 & 1.5 \\
Confidence level (95\%) & 2.1 & 2.3 & 3.0 \\
\hline
\end{tabular}

$M X$ mammography, US ultrasound, CEDM contrast-enhanced digital mammography
22 patients with suspect abnormalities found on conventional mammography or ultrasound [10]. The results showed the ability of temporal CEDM to show cancers and suggested a potential to identify cancers in dense breasts. Dromain et al. also concluded from a 20-patient study that temporal CEDM has the potential to depict angiogenesis [11]. A more extended temporal CEDM study on 75 patients with 85 lesions compared the performance of conventional mammography alone versus temporal CEDM as an adjunct to conventional mammography [12]. The results indicated an improvement in the sensitivity and specificity when adding temporal CEDM to the conventional mammography. However, several limitations affect temporal CEDM: the long examination and breast compression time contribute to patient discomfort and increases the probability of patient motion, generating artefacts on the subtracted images; moreover, only one view per breast can be acquired for a single injection of contrast medium; in addition to this, there has been no proof that the information provided by the contrast agent uptake kinetics is clinically useful. In 
Table 6 Assessment of the number of lesions by each imaging technique in multifocal carcinomas

$M X$ mammography, US ultrasound, $C E D M$ contrastenhanced digital mammography IDC Invasive ductal carcinoma, ILC Invasive lobular carcinoma

\begin{tabular}{|c|c|c|c|c|c|c|}
\hline \multirow[t]{2}{*}{ Patients } & \multicolumn{2}{|l|}{ Histology } & \multirow[t]{2}{*}{ Physical examination } & \multirow[t]{2}{*}{ MX } & \multirow[t]{2}{*}{ US } & \multirow[t]{2}{*}{ CEDM } \\
\hline & No. of foci & Type & & & & \\
\hline 12 & 2 & IDC & 0 & 1 & 0 & 2 \\
\hline 13 & 2 & IDC & 1 & 2 & 2 & 2 \\
\hline 52 & 2 & ILC & 1 & 1 & 2 & 2 \\
\hline 68 & 2 & IDC & 0 & 2 & 1 & 2 \\
\hline 72 & 2 & IDC & 1 & 1 & 1 & 2 \\
\hline 79 & 3 & IDC & 1 & 0 & 1 & 3 \\
\hline 93 & 3 & IDC+DCIS & 0 & 3 & 3 & 3 \\
\hline 96 & 2 & ILC & 2 & 2 & 2 & 2 \\
\hline 98 & 2 & IDC & 0 & 1 & 1 & 2 \\
\hline 114 & 3 & IDC & 1 & 3 & 2 & 3 \\
\hline Total & 23 & & 7 & 16 & 15 & 23 \\
\hline
\end{tabular}

Diekmann et al.'s study, it appeared that the diagnostically relevant information was mainly given by the morphology and intensity of the contrast agent uptake (12). No correlation could be found between the contrast enhancement pattern and the malignant nature of the lesion. In Dual-Energy CEDM, to date, only one feasibility study has been performed on 26 patients by Lewin et al. [13]. His results demonstrated enhancement in all of the 13 invasive cancers included in the study, with strong uptake in 11 of these cases. A case of ductal carcinoma in situ showed weak enhancement in the duct. Among the remaining 12 benign cases, 8 displayed no enhancement, 2 enhanced diffusely and 2 demonstrated weak focal enhancements. No quantification of the performance of the method was performed in this study, because of the restricted number of recruited patients.

The present study confirms the good diagnostic accuracy of CEDM for the detection of breast carcinoma, which was here superior to mammography alone and to mammography interpreted in association with ultrasound. CEDM demonstrated contrast agent uptake in most of the malignant lesions. Compared with mammography alone, CEDM significantly increased the sensitivity without a loss in specificity. CEDM also allowed a gain for the negative predictive value with a significant reduction in the false negatives. Compared with the standard of care for diagnostic procedures (i.e. mammography associated with breast ultrasound), CEDM had a better diagnostic accuracy mainly due to improved specificity, and better positive and negative predictive values. CEDM has the advantage of being reproducible without operator dependency. Moreover, CEDM is a fast imaging technique and subtracted images have a direct correlation with conventional mammograms.

Contrast-enhanced digital mammography can be performed by using a current digital mammography system with some
Fig. 6 Invasive ductal carcinoma in a 58-year-old woman with a normal physical examination. The screening mammography with mediolateral view (a) and cranio-caudal view (b) shows uncertain distortion seen only on a single view (arrow). The subtracted dual-energy CEDM images on the mediolateral view (c) and cranio-caudal view (d) clearly depict an obvious mass in the outer and superior quadrant with irregular margin highly suggestive of a malignant lesion. One can note on subtracted CEDM images a whiter region near the breast edge images corresponding to a scattered radiation artifact
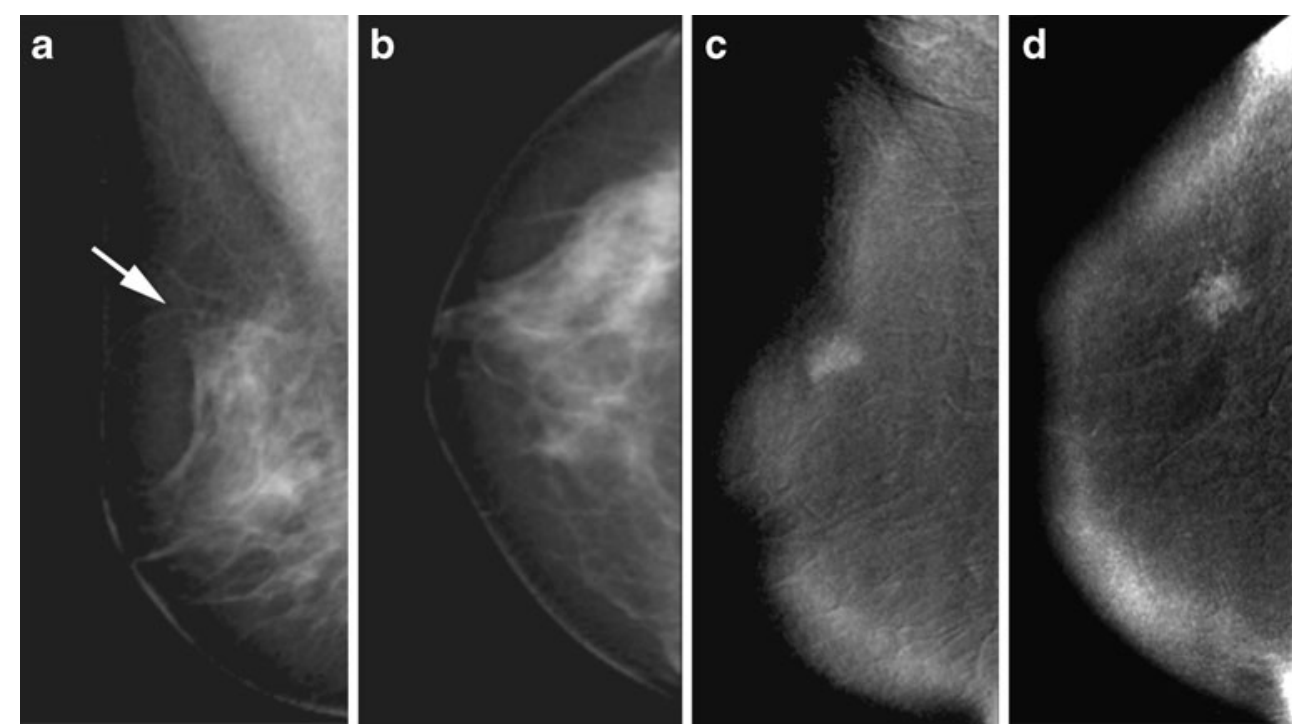

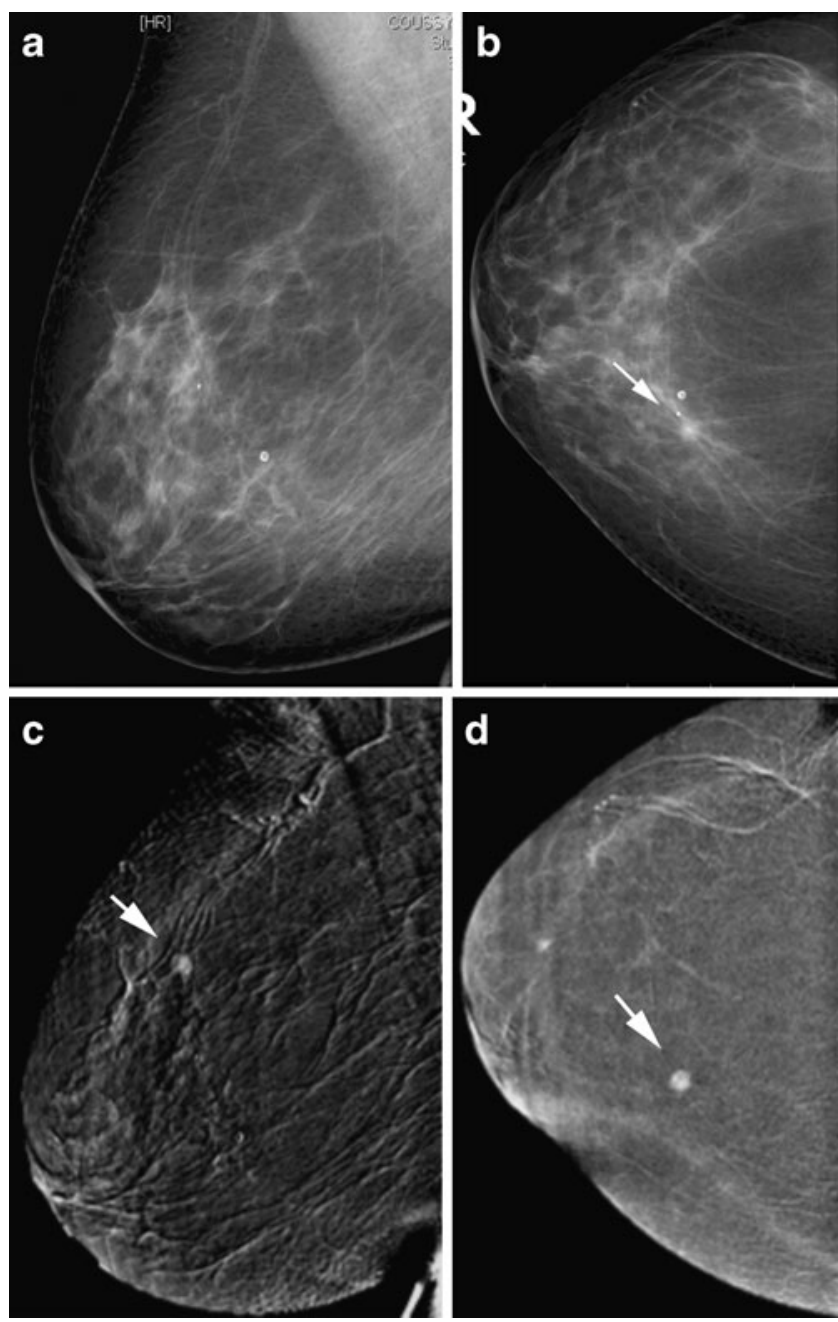

Fig. 7 Small invasive ductal carcinoma in a 65-year-old woman. The screening mammography with the mediolateral view (a) and the cranio-caudal view (b) shows a small opacity only visible on the CC view. The subtracted dual-energy CEDM images on the mediolateral view (c) and the cranio-caudal view (d) readily depict a small enhancing nodule corresponding to a $5-\mathrm{mm}$ invasive ductal carcinoma

specific software and hardware adaptations for acquisition and image processing. CEDM is of interest in the non-screening setting and has the potential to increase the cancer detection rate, to improve staging and to improve the selection of patients for biopsy. At present, breast MRI is considered to be the most sensitive imaging technique for breast cancer detection and assessment of the extent of disease [19-22]. However breast MRI is expensive and not widely available. Moreover, the quality of breast MRI may vary significantly across practices. The present study hints that, similar to breast MRI, CEDM could be of particular interest for the assessment of the extent of disease. Indeed, our study has shown that CEDM allowed an accurate lesion size evaluation and detected multifocal breast cancers in all cases. The dual-energy technique offers the possibility of imaging both breasts in two views (for example CC and MLO) during a single injection of contrast medium and so to perform a bilateral CEDM examination. Another clinical indication for CEDM could be "problem solving" in the case of equivocal mammography and ultrasound assessments (Figs. 6 and 7). In this potential indication CEDM has the advantage of being a fast imaging technique with immediate availability in the mammography suite without a new appointment and without loss of time. It could be particularly of interest in dense breasts where the sensitivity of mammography is lower (Fig. 8). Even if slightly higher than that of MX+US the specificity
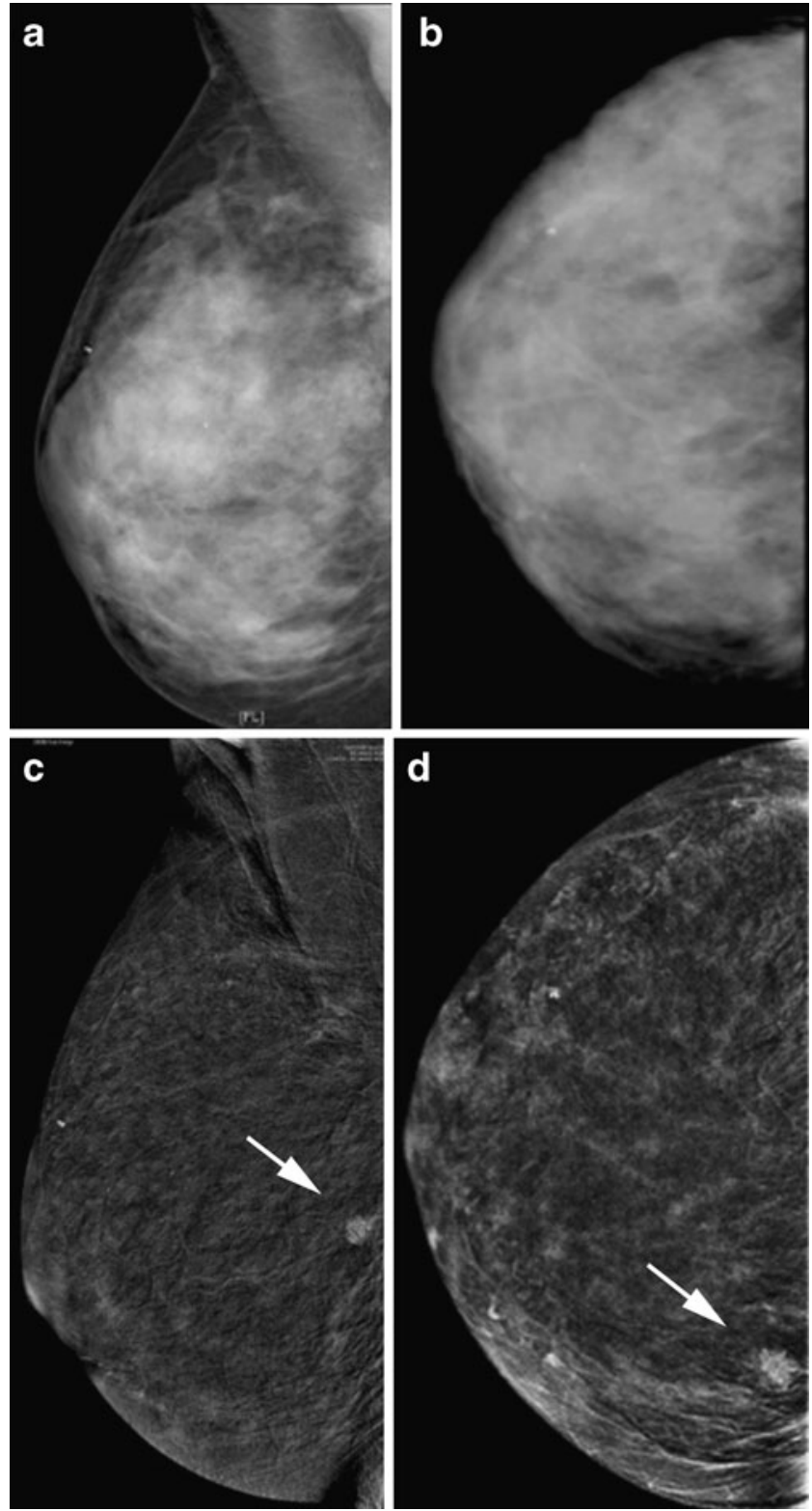

Fig. 8 Invasive ductal carcinoma in a 50-year-old patient. Screening mammography with the mediolateral view (a) and the cranio-caudal view (b) shows very dense breasts with no obvious abnormal findings. The subtracted dual-energy CEDM images on the mediolateral view (c) and the cranio-caudal view (d) clearly demonstrate an irregular nodule in the deep part of the breast 
of CEDM was not optimal and some benign lesions could depict a significant enhancement such as fibroadenomas, phylloid tumours, intraductal papillomas, radial scars and cytosteatonecrosis. Of the 120 patients, CEDM depicted only 4 cases of enhancement corresponding to adenosis and fibrocystic mastopathy. In most of cases the morphology of these lesions analysed on low-energy images (equivalent to a conventional mammogram) in conjunction with the analysis of the subtracted images were consistent with the benignity.

On the recombined dual energy CEDM images, one can note a whiter region near the breast edge images impacting negatively the analysis of peripheral area of the breast (Fig. 6). This artifact can be explained by the presence of scattered radiation, which is non-uniform throughout the breast and has different characteristics at low and high energy, and by the decrease of the breast thickness towards the edge. By correlating the information seen on the recombined image and the low-energy image (similar to a conventional mammogram), this whiter region can be usually recognized as an artefact and not misinterpreted as iodine uptake. Another cause of artifacts observed in subtracted images is patient motion. However, because of the short interval time between the low and high energy images this type of artifacts is much less frequent than with the temporal CEDM technique (Fig. 7).

The present study had some limitations. Interpretation of ultrasound examinations was based on the review of static ultrasound images recorded on the PACS system and thus were dependent on the initial operators. Also, only one reader reviewed all images for each imaging technique. We believe that these preliminary results should be confirmed by a multireader review. The study has focused on invasive cancers because of the exclusion criteria of isolated clusters of microcalcifications. However, it reflects the potential clinical application of CEDM.

The results of this preliminary clinical trial suggest that dual-energy CEDM has a higher diagnostic accuracy for the detection of breast carcinoma compared with mammography alone and with mammography interpreted in association with ultrasound. CEDM may be useful for the assessment of the extent of disease as well as the clarification of equivocal lesions. Development of this technique should be associated with the ability to perform CEDM-guided needle localisation or biopsy. These results encourage further investigations to define the place of CEDM among the other breast imaging methods in particular in comparison to breast MRI.

Acknowledgements This work was supported by a grant from the Ministère Français de la Jeunesse, de l'Education et de la Recherche.

Serge Muller is employee of GE Healthcare. His contribution in the present paper was limited to lead the development of the experimental device used during the clinical study.

\section{References}

1. Duffy SW (2006) Reduction in breast cancer mortality from organized service screening with mammography: further confirmation with external data. Cancer Epidemiol Biomarkers Prev 15:45-51

2. Tabar L, Yen MF, Vitak B et al (2003) Mammography service screening and mortality in breast cancer patients: 20 years follow-up before and after introduction of screening. Lancet 361:1405-1410

3. Otto SJ, Fracheboud J, Looman CW et al (2003) Initiation of population-based mammography screening in Dutch municipalities and effect on breast cancer mortality: a systematic review. Lancet 361:411-417

4. Rosenberg RD, Hunt WC, Williamson MR et al (1998) Effect of age, breast density, ethnicity and estrogen replacement therapy on screening mammographic sensitivity and cancer stage at diagnosis: review of 183, 134 screening mammograms in Albuquerque. New Mexico Radiol 209:511518

5. Kolb TM, Lichy J, Newhouse JH (2002) Comparison of the performance of screening mammography physical examination, and breast US and evaluation of factors that influence them: an analysis of 27, 825 patient evaluations. Radiology 255:165-175

6. Kolb TM, Lichy J, Newhouse JH (1998) Occult cancer in women with dense breasts: detection with screening US - diagnostic yield and tumor characteristics. Radiology 207:191-199

7. Hagay C, Cherel P, de Maulmont CE et al (1996) Contrastenhanced CT: value for diagnosing local breast cancer recurrence after conservative treatment. Radiology 200:631638

8. Chang CH, Nesbit DE, Fischer DR et al (1982) Computed tomographic mammography using a conventional body scanner. AJR Am J Roentgenol 138:553-558

9. Peters NHGM, Borel Rinkes IHM, Zuithof NPA et al (2008) Meta-analysis of MR imaging in the diagnosis of breast lesions. Radiology 246(1):116-124

10. Jong RA, Yaffe MJ, Skarpathiotakis M et al (2003) Contrast enhanced digital mammography: initial clinical experience. Radiology 228:842-850

11. Dromain C, Balleyguier C, Muller S et al (2006) Evaluation of tumor angiogenesis of breast carcinoma using contrastenhanced digital mammography. AJR Am J Roentgenol 187:528-537

12. Diekmann F, Marx C, Jong R et al (2007) Diagnostic accuracy of contrast-enhanced digital mammography as an adjunct to mammography. Abstract. Eur Radiol 17:174

13. Lewin JM, Isaacs PK, Vance V et al (2003) Dual-energy contrastenhanced subtraction mammography: feasibility. Radiology 229:261-268

14. Skarpathiotakis M, Yaffe MJ, Bloomquist AK et al (2002) Development of contrast digital mammography. Med Phys 29:2419-2426

15. Puong S, Bouchevreau X, Patoureaux F et al (2007) Dual-energy contrast enhanced digital mammography using a new approach for breast tissue cancelling. Proceedings of SPIE Medical Imaging 6510:65102H

16. Xizeng Wu, Barnes GT, Tucker DM (1991) Spectral dependence of glandular tissue dose in screen-film mammography. Radiology 179:144-148

17. Xizeng Wu, Gingold EL, Barnes GT et al (1994) Normalized average glandular dose in molybdenum target-rhodium filter and rhodium molybdenum target—rhodium filter. Radiology 193:83898 
18. Boone JM (2002) Normalized glandular dose ( $\mathrm{DgN})$ coefficients for arbitrary X-ray spectra in mammography: computer-fit values of Monte Carlo derived data. Med Phys 29:869-875

19. Orel SG, Schnall MD, Powell CM, Hochman MG, Solin LJ, Fowble BL, Torosian MH, Rosato EF (1995) Staging of suspected breast cancer: effect of MR imaging and MR-guided biopsy. Radiology 196:115-122

20. Berg WA, Gutierrez L, NessAiver MS et al (2004) Diagnostic accuracy of mammography, clinical examination, ultrasound, and
MR imaging in pre-operative assessment of breast cancer. Radiology 233:830-849

21. Lehman CD, Gatsonis C, Kuhl CK et al (2007) ACRIN trial 6667 investigators group. MRI evaluation of the contralateral breast in women with recently diagnosed breast cancer. N Engl J Med 356:1295-1303

22. Hollingsworth AB, Stough RG, O'Dell CA et al (2008) Breast magnetic resonance imaging for preoperative locoregional staging. Am J Surg 196:389-397 